\title{
Article
}

\section{Curious Generalized Fibonacci Numbers}

\author{
Jose L. Herrera ${ }^{1, * \mathbb{C}}$, Jhon J. Bravo ${ }^{1}$ and Carlos A. Gómez ${ }^{2}$ \\ 1 Departamento de Matemáticas, Universidad del Cauca, Popayán 190003 ,Colombia; jbravo@unicauca.edu.co \\ 2 Departamento de Matemáticas, Universidad del Valle, Cali 25360 , Colombia; \\ carlos.a.gomez@correounivalle.edu.co \\ * Correspondence: joseherrera@unicauca.edu.co
}

Citation: Herrera, J.L.; Bravo, J.J.; Gómez , C.A. Curious Generalized Fibonacci Numbers. Mathematics 2021, 9, 2588. https://doi.org/10.3390/ math9202588

Received: 23 August 2021 Accepted: 19 September 2021 Published: 15 October 2021

Publisher's Note: MDPI stays neutral with regard to jurisdictional claims in published maps and institutional affiliations.

Copyright: (c) 2021 by the authors. Licensee MDPI, Basel, Switzerland. This article is an open access article distributed under the terms and conditions of the Creative Commons Attribution (CC BY) license (https:// creativecommons.org/licenses/by/ $4.0 /)$.

\begin{abstract}
A generalization of the well-known Fibonacci sequence is the $k$-Fibonacci sequence whose first $k$ terms are $0, \ldots, 0,1$ and each term afterwards is the sum of the preceding $k$ terms. In this paper, we find all $k$-Fibonacci numbers that are curious numbers (i.e., numbers whose base ten representation have the form $a \cdots a b \cdots b a \cdots a)$. This work continues and extends the prior result of Trojovský, who found all Fibonacci numbers with a prescribed block of digits, and the result of Alahmadi et al., who searched for $k$-Fibonacci numbers, which are concatenation of two repdigits.
\end{abstract}

Keywords: generalized Fibonacci number; repdigit; linear form in logarithms; reduction method

MSC: 11B39; 11J86

\section{Introduction}

Given a couple of non-negative integers $\ell$ and $m$, we shall define the $(\ell, m)$-curious number as a natural number with the following base ten representation

$$
\underbrace{a \cdots a}_{\ell} \underbrace{b \cdots b}_{m} \underbrace{a \cdots a}_{\ell}
$$

where $a$ and $b$ are integers such that $a, b \in\{0,1, \ldots, 9\}$. The first curious numbers are

$$
\mathcal{C}=\{0,1,2,3,4,5,6,7,8,9,11,22,33,44,55,66,77,88,99,101,111,121,131,141, \ldots\},
$$

and this matches with the sequence A335779 in Sloane's Encyclopedia [1]. Few properties of curious numbers are currently known. For instance, Borade and Mayle in [2] determined all curious number that are perfect squares. Note that a $(0, m)$-curious number is not more than a repdigit, i.e., a positive integer with only one distinct digit in its decimal representation.

However, many papers have been written on Diophantine equations involving repdigits and terms of certain linear recurrence sequences. It should be mentioned that Luca [3] in 2000 showed that 55 and 11 are the largest repdigits in the Fibonacci and Lucas sequences, respectively. Since then, this result has been generalized and extended in various directions. For example, Erduvan and Keskin [4] found all repdigits expressible as products of two Fibonacci or Lucas numbers. Faye and Luca [5] looked for repdigits in the usual Pell sequence; using some elementary methods, they concluded that there are no Pell numbers larger than 10 that are repdigits. We also mention the work of Normenyo, Luca and Togbé [6] who found all repdigits expressible as sums of three Pell numbers. Shortly afterwards, they extended their work to four Pell numbers [7].

The Fibonacci sequence has been generalized in many ways. Here, we consider, for an integer $k \geq 2$, the $k$-Fibonacci sequence $F^{(k)}=\left(F_{n}^{(k)}\right)_{n \geq-(k-2)}$ defined by the recurrence relation

$$
F_{n}^{(k)}=F_{n-1}^{(k)}+\cdots+F_{n-k}^{(k)} \text { for all } n \geq 2,
$$


with initial values $F_{i}^{(k)}=0$ for $i=2-k, \ldots, 0$, and $F_{1}^{(k)}=1$. We call $F_{n}^{(k)}$ the $n$th $k$-Fibonacci number. The Fibonacci numbers are obtained for $k=2$, while when, for example, $k=3$, the resulting sequence is a tribonacci sequence.

Diophantine problems involving $k$-Fibonacci numbers and repdigits were recently an active research field in number theory. For example, a conjecture (proposed by Marques [8]) about repdigits in $k$-Fibonacci sequences was proved by Bravo-Luca [9]. Alahmadi et al. [10] generalized recently the results mentioned above by showing that only repdigits with at least two digits as a product of $\ell$ consecutive $k$-Fibonacci numbers occur only for $(k, \ell)=(2,1),(3,1)$, extending the works [11,12], which dealt with the particular cases of Fibonacci and Tribonacci numbers. See [13] for a problem involving repdigits in generalized pell sequences. In addition, Bravo-Luca [14] found all repdigits, which are sums of two $k$-Fibonacci numbers (see [15] for a product version). Problems concerning powers of two and coincidences in generalized Fibonacci numbers can be found in $[16,17]$. Finally, Alahmadi et al. [18] determined all $k$-Fibonacci numbers that are concatenations of two repdigits, while Trojovský [19] found all Fibonacci numbers with a prescribed block of digits.

In this paper, we determine all curious numbers, which are $k$-Fibonacci numbers, i.e.,

$$
F_{n}^{(k)}=\underbrace{a \cdots a}_{\ell} \underbrace{b \cdots b}_{m} \underbrace{a \cdots a}_{\ell}
$$

which continues and extends the works in [18,19]. More precisely, we solve the Diophantine equation as follows:

$$
F_{n}^{(k)}=\frac{1}{9}\left(a \cdot 10^{2 \ell+m}-(a-b) \cdot 10^{\ell+m}+(a-b) \cdot 10^{\ell}-a\right)
$$

in positive integers $n, k, m, \ell, a$ and $b$ with $k \geq 2, a, b \in\{0,1, \ldots, 9\}$ and $a \neq b$.

Before presenting our main theorem, it is important to mention that in Equation (1), we assumed $\ell, m \geq 1$ and $a \neq b$ since otherwise, the problem reduces to finding all $k$-Fibonacci numbers that are repdigits or concatenations of two repdigits; these problems have been already solved in [9,18] (see also [20]). In addition, note that when $a=0$, our problem also reduces to determining all $k$-Fibonacci numbers that are concatenations of two repdigits. Thus, throughout this paper we also assume that $a \geq 1$. Our result is the following.

Theorem 1. The only curious generalized Fibonacci number is $F_{11}^{(5)}=464$.

As an immediate consequence of Theorem 1, we have the following corollary.

Corollary 1. There are no curious numbers that are powers of two.

\section{Preliminary Results}

In this section, we present some basic properties of the $k$-Fibonacci sequence and give some important estimations needed for the sequel. Additionally, we present a lower bound for a nonzero linear form in logarithms of algebraic numbers and state two reduction lemmas, which will be the key tool used in this paper to reduce the upper bounds. All these facts will be used in the proof of Theorem 1.

\subsection{On k-Fibonacci Numbers}

The first direct observation about the $k$-Fibonacci sequence is that the first $k+1$ non-zero terms in $F^{(k)}$ are powers of two, namely, the following:

$$
F_{n}^{(k)}=2^{\max \{0, n-2\}} \quad \text { for all } \quad 1 \leq n \leq k+1,
$$


while the next term is $F_{k+2}^{(k)}=2^{k}-1$. In fact, the inequality (see [16])

$$
F_{n}^{(k)}<2^{n-2} \text { holds for all } n \geq k+2 .
$$

Next, $F^{(k)}$ is a linear recurrence sequence of the following characteristic polynomial:

$$
\Psi_{k}(z)=z^{k}-z^{k-1}-\cdots-z-1 .
$$

The classic study of linear recurrence sequences (see [21]) is based on knowledge of the roots of their characteristic polynomials. While studying the roots of $\Psi_{k}(z)$, it is usual to work with the shifted polynomial as follows:

$$
\psi_{k}(z)=(z-1) \Psi_{k}(z)=z^{k+1}-2 z^{k}+1 .
$$

Except for the extra root at $z=1, \psi_{k}(z)$ has the same roots as $\Psi_{k}(x)$. By Descartes' rule of signs, the polynomial $\Psi_{k}(z)$ has exactly one positive real root, for example, $z=\alpha(k)$. Since $\Psi_{k}(1)=1-k$ and $\Psi_{k}(2)=1$, it follows that $\alpha(k) \in(1,2)$. In fact, it is known that $2\left(1-2^{-k}\right)<\alpha(k)<2$ (see [22] (Lemma 2.3) or [23] (Lemma 3.6)). Thus, $\alpha(k)$ approaches 2 as $k$ tends to infinity. To simplify the notation, we shall omit the dependence on $k$ of $\alpha$.

Miles [24] showed that the roots of $\Psi_{k}(z)$ are distinct, and the remaining $k-1$ roots of $\Psi_{k}(z)$ different from $\alpha$ lie inside the unit disk. He showed this by reducing the equation $\Psi_{k}(z)=0$ to a form where Rouch's theorem could be applied. This fact was reproved by Miller [25] by an elementary argument. In particular, $\alpha$ is a Pisot number of degree $k$, and $\Psi_{k}(z)$ is an irreducible polynomial over $\mathbb{Q}[z]$.

We consider for $k \geq 2$, the function $f_{k}(z):=(z-1) /(2+(k+1)(z-2))$. With this notation, Dresden and Du proved in [26] the following:

$$
\left|F_{n}^{(k)}-f_{k}(\alpha) \alpha^{n-1}\right|<\frac{1}{2}
$$

which for all $n \geq 1$ and $k \geq 2$. From (4), we can write the following:

$$
F_{n}^{(k)}=f_{k}(\alpha) \alpha^{n-1}+e_{k}(n) \quad \text { where }\left|e_{k}(n)\right|<1 / 2 .
$$

Furthermore, we obtain the following:

$$
\left.1 / 2 \leq f_{k}(\alpha) \leq 3 / 4 \text { and } \mid f_{k}\left(\alpha_{i}\right)\right) \mid<1 \text { for } i=2, \ldots, k,
$$

which hold for all $k \geq 2$, where $\alpha=: \alpha_{1}, \alpha_{2}, \ldots, \alpha_{k}$ are all the zeros of $\Psi_{k}(z)$. So, by computing norms from $\mathbb{Q}(\alpha)$ to $\mathbb{Q}$, we see that the number $f_{k}(\alpha)$ is not an algebraic integer. Proofs for this fact and inequalities (6) can be found in [27].

Additionally, it was proved in [9] the following:

$$
\alpha^{n-2} \leq F_{n}^{(k)} \leq \alpha^{n-1} \text { holds for all } n \geq 1 \text { and } k \geq 2 .
$$

We finish this subsection with the following estimate, due to Bravo, Gómez and Luca [28], which will be one of the key points in addressing the large values of $k$ (see also [29]).

Lemma 1. Let $k \geq 2$ and suppose that $r<2^{k / 2}$. Then, the following holds:

$$
F_{r}^{(k)}=2^{r-2}\left(1+\zeta_{f}\right) \text { where }\left|\zeta_{f}\right|<\frac{1}{2^{k / 2}} .
$$

\subsection{Linear Forms in Logarithms}

We need to use a Baker-type lower bound for a nonzero linear form in logarithms of algebraic numbers. We begin by recalling some basic notions from algebraic number theory. 
Let $\eta$ be an algebraic number of degree $d$ over $\mathbb{Q}$ with minimal primitive polynomial over the integers $m(z):=a_{0} \prod_{i=1}^{d}\left(z-\eta^{(i)}\right) \in \mathbb{Z}[z]$, where the leading coefficient $a_{0}$ is positive and the $\eta^{(i)}$ 's are the conjugates of $\eta$. The logarithmic height of $\eta$ is given by the following:

$$
h(\eta):=\frac{1}{d}\left(\log a_{0}+\sum_{i=1}^{d} \log \max \left\{\left|\eta^{(i)}\right|, 1\right\}\right) .
$$

In order to illustrate this, we can use the facts that the minimal primitive polynomial of $\alpha$ is $\Psi_{k}(z), \mathbb{Q}(\alpha)=\mathbb{Q}\left(f_{k}(\alpha)\right)$ and that $\left|f_{k}\left(\alpha^{(i)}\right)\right| \leq 1$ for all $i=1, \ldots, k$ and $k \geq 2$ (see (6)), to prove the following:

$$
h(\alpha)=(\log \alpha) / k<(\log 2) / k \text { and } h\left(f_{k}(\alpha)\right)<2 \log k \text { for all } k \geq 2 .
$$

See [27] for further details of the proof of (8). In addition, if $\eta=p / q$ is a rational number with $\operatorname{gcd}(p, q)=1$ and $q>0$, then $h(\eta)=\log \max \{|p|, q\}$. Finally, the following properties of $h(\cdot)$ are used in the next sections:

$$
\begin{aligned}
h(\eta \pm \gamma) \leq h(\eta)+h(\gamma)+\log 2 ; & h\left(\eta \gamma^{ \pm 1}\right) \leq h(\eta)+h(\gamma) \\
h(\eta) & =h\left(\eta^{(i)}\right), \quad i=2, \ldots, k ; \quad h\left(\eta^{s}\right)=|s| h(\eta) \quad(s \in \mathbb{Z}) .
\end{aligned}
$$

As a consequence of the above properties, one can easily deduce the next lemma.

Lemma 1. Let $k \geq 2$ and $s \neq 0$ be the integers and suppose that $|s| \leq 10^{\varepsilon}$ for some integer $\varepsilon \geq 1$. Then, we have the following:

$$
h\left(9 f_{k}(\alpha) s^{-1}\right)<\varepsilon \log 10+2 \log k
$$

Furthermore, if $\varepsilon=1$, then we have the following:

$$
h\left(9 f_{k}(\alpha) s^{-1}\right)<6 \log k .
$$

Our main tool is the following lower bound for a non-zero linear form in logarithms of algebraic numbers, due to Matveev [30].

Theorem 1 (Matveev's theorem). Let $\mathbb{K}$ be a number field of degree $D$ over $\mathbb{Q}, \gamma_{1}, \ldots, \gamma_{t}$ be positive real numbers of $\mathbb{K}$, and $b_{1}, \ldots, b_{t}$ rational integers. Put $\Lambda:=\gamma_{1}^{b_{1}} \cdots \gamma_{t}^{b_{t}}-1$ and $B \geq \max \left\{\left|b_{1}\right|, \ldots,\left|b_{t}\right|\right\}$. Let $A_{i} \geq \max \left\{D h\left(\gamma_{i}\right),\left|\log \gamma_{i}\right|, 0.16\right\}$ be real numbers for $i=1, \ldots, t$. Then, assuming that $\Lambda \neq 0$, we have the following:

$$
|\Lambda|>\exp \left(-1.4 \times 30^{t+3} \times t^{4.5} \times D^{2}(1+\log D)(1+\log B) A_{1} \cdots A_{t}\right) .
$$

\subsection{Reduction Tools}

To lower the bounds arising from applying Theorem 1, we will use a result from the theory of continued fractions. The following lemma is a slight variation of a result, due to Dujella and Pethő [31]. We shall use the version given by Bravo, Gómez and Luca (see [27] [Lemma 1]).

Lemma 2. Let $\tau$ be an irrational number, and let $A, B, \mu$ be real numbers with $A>0$ and $B>1$. Assume that $M$ is a positive integer. Let $p / q$ be a convergent of the continued fraction of $\tau$ such that $q>6 M$ and put $\epsilon:=\|\mu q\|-M\|\tau q\|$, where $\|\cdot\|$ denotes the distance from the nearest integer. If $\epsilon>0$, then there is no solution of the inequality

$$
0<|u \tau-v+\mu|<A B^{-w}
$$

in positive integers $u, v$ and $w$ with $u \leq M$ and $w \geq \log (A q / \epsilon) / \log B$. 
The above lemma cannot be applied when $\mu$ is an integer linear combination of 1 and $\tau$ since then, $\epsilon<0$. In this case, we use the following nice property of continued fractions (see Theorem 8.2.4 and top of page 263 in [32]).

Lemma 3. Let $p_{i} / q_{i}$ be the convergent of the continued fraction $\left[a_{0}, a_{1}, \ldots\right]$ of the irrational number $\gamma$. Let $M$ be a positive integer and put $a_{M}:=\max \left\{a_{i} \mid 0 \leq i \leq N+1\right\}$ where $N \in \mathbb{N}$ is such that $q_{N} \leq M<q_{N+1}$. If $x, y \in \mathbb{Z}$ with $x>0$, then we have the following:

$$
|x \gamma-y|>\frac{1}{\left(a_{M}+2\right) x} \quad \text { for all } \quad x \leq M .
$$

We finish with the following simple facts concerning the exponential function. We list it as a lemma for further reference, and its proof can be found in [33].

Lemma 4. For any non-zero real number $x$, we have the following:

(a) $0<x<\left|e^{x}-1\right|$.

(b) If $x<0$ and $\left|e^{x}-1\right|<1 / 2$, then $|x|<2\left|e^{x}-1\right|$.

\section{Proof Theorem 1}

Assume throughout that $(n, k, a, b, \ell, m)$ is a solution of Equation (1). First, we note that $n \leq 3$ is impossible since $F_{n}^{(k)}$ must have at least 3 digits in its decimal representation. Thus, we assume $n \geq 4$. We now want to establish a relationship between the variables of (1). For this purpose, we use Equations (1), (3) and (7) to obtain the following:

$$
10^{2 \ell+m-1}<F_{n}^{(k)} \leq 2^{n-2} \quad \text { and } \quad \alpha^{n-2} \leq F_{n}^{(k)}<10^{2 \ell+m}
$$

giving the following:

$$
2 \ell+m<(n-2)\left(\frac{\log 2}{\log 10}\right)+1 \text { and } n-2<(2 \ell+m)\left(\frac{\log 10}{\log \alpha}\right) .
$$

In particular, we have the following:

$$
(2 \ell+m)+2<n<6(2 \ell+m) \text { holds for all } n \geq 4 .
$$

\subsection{Powers of 2 Which Are Curious Numbers}

Assuming $4 \leq n \leq k+1$ and taking into account (2), we can rewrite Equation (1) as follows:

$$
a \cdot 10^{2 \ell+m}-(a-b) \cdot 10^{\ell+m}+(a-b) \cdot 10^{\ell}-9 \cdot 2^{n-2}=a .
$$

Since $\ell<n-2$ by (10), it follows from (11) that $2^{\ell} \mid a$ and so $\ell \leq 3$. We now use (11) once again to obtain the following:

$$
a \cdot 10^{2 \ell+m}-(a-b) \cdot 10^{\ell+m}-9 \cdot 2^{n-2}=a-(a-b) \cdot 10^{\ell} \in \mathcal{R},
$$

where $\mathcal{R}:=([-8991,0) \cup(0,8001]) \cap \mathbb{Z}$. Since the largest 2 -adic valuation of integers of the interval $\mathcal{R}$ is 7 , by (12), we obtain $\ell+m \leq 7$. So, $m \leq 6$. Finally, a numerical check with Mathematica reveals that Equation (1) has no solutions in the following range (see Appendix A):

$$
4 \leq n \leq k+1, \quad 1 \leq \ell \leq 3, \quad 1 \leq m \leq 6, \quad 1 \leq a \leq 9 \quad \text { and } \quad 0 \leq b \leq 9 .
$$

Thus, from now on, we suppose that $n \geq k+2$. 


\subsection{Bounding $n$ in Terms of $k$}

In this subsection, we want to find an upper bound for $n$ in terms of $k$. To do this, we use (5) and rewrite (1) in two different forms, namely, the following:

$$
\begin{gathered}
9 f_{k}(\alpha) \alpha^{n-1}-a \cdot 10^{2 \ell+m}=-9 e_{k}(n)-(a-b) \cdot 10^{\ell+m}+(a-b) \cdot 10^{\ell}-a, \\
9 f_{k}(\alpha) \alpha^{n-1}-10^{\ell+m} X=-9 e_{k}(n)+(a-b) \cdot 10^{\ell}-a,
\end{gathered}
$$

where we have put $X:=a \cdot 10^{\ell}-(a-b)$. For future calculations, it will be important to note the following:

$$
1 \leq X \leq 10^{\ell+1} .
$$

We now take absolute value in relations given by (13); doing some straightforward calculations, we obtain the following:

$$
\begin{gathered}
\left|9 f_{k}(\alpha) \alpha^{n-1}-a \cdot 10^{2 \ell+m}\right|<11 \cdot 10^{\ell+m}, \\
\left|9 f_{k}(\alpha) \alpha^{n-1}-10^{\ell+m} X\right|<11 \cdot 10^{\ell} .
\end{gathered}
$$

Dividing both sides of each one of the above inequalities (15) by $a \cdot 10^{2 \ell+m}$ and $10^{\ell+m} X$, respectively, and rearranging some terms, we obtain the following:

$$
\begin{gathered}
\left|\alpha^{n-1} \cdot 10^{-(2 \ell+m)} \cdot\left(\frac{9 f_{k}(\alpha)}{a}\right)-1\right|<11 / 10^{\ell}, \quad \text { and } \\
\left|\alpha^{n-1} \cdot 10^{-(\ell+m)} \cdot\left(\frac{9 f_{k}(\alpha)}{X}\right)-1\right|<11 / 10^{m} .
\end{gathered}
$$

At this point, we claim that the left-hand sides of (16) and (17) are not zero. Indeed, if these were zero, we would then obtain the following, respectively:

$$
a \cdot 10^{2 \ell+m}=9 f_{k}(\alpha) \alpha^{n-1} \text { and } 10^{\ell+m} X=9 f_{k}(\alpha) \alpha^{n-1},
$$

Conjugating with an automorphism $\sigma$ of the Galois group of $\Psi_{k}(x)$ over $\mathbb{Q}$ such that $\sigma(\alpha)=\alpha_{i}$ for some $i>1$, taking absolute values and using the fact that $\left|9 f_{k}\left(\alpha_{i}\right) \alpha_{i}^{n-1}\right|<9$, we obtain the following, respectively:

$$
a \cdot 10^{2 \ell+m}<9 \text { and } 10^{\ell+m} X<9,
$$

However, these lead to a contradiction since the following are true:

$$
a \cdot 10^{2 \ell+m} \geq 10^{3} \text { and } 10^{\ell+m} X \geq 10^{2} .
$$

We shall now apply Matveev's theorem on inequalities (16) and (17) (in that order). To do this, we take the following parameters:

$$
\begin{gathered}
t:=3, \quad \gamma_{1}:=\alpha, \quad \gamma_{2}:=10, \quad \gamma_{3,1}:=9 f_{k}(\alpha) / a, \quad \gamma_{3,2}:=9 f_{k}(\alpha) / X, \\
b_{1}:=n-1, \quad b_{2,1}:=-(2 \ell+m), \quad b_{2,2}:=-(\ell+m), \quad b_{3}:=1 .
\end{gathered}
$$

The real number field containing $\gamma_{1}, \gamma_{2}, \gamma_{3,1}, \gamma_{3,2}$ is $\mathbb{K}:=\mathbb{Q}(\alpha)$. From this and (10), we can take $D:=[\mathbb{K}: \mathbb{Q}]=k$ and $B:=n$ in any application of Matveev's theorem.

On the other hand, since $h\left(\gamma_{1}\right)<(\log 2) / k($ by $(8))$ and $h\left(\gamma_{2}\right)=\log 10$, we can always take $A_{1}:=\log 2$ and $A_{2}:=k \log 10$. Furthermore, from Lemma 1 and (14) we obtain the following:

$$
\begin{gathered}
h\left(\gamma_{3,1}\right)<6 \log k \\
h\left(\gamma_{3,2}\right)<2 \log k+(\ell+1) \log 10 .
\end{gathered}
$$




\subsubsection{An Inequality for $\ell$ in Terms of $k$}

In order to apply Matveev's theorem on (16) with the parameters $\gamma_{1}, \gamma_{2}$ and $\gamma_{3,1}$, we take $A_{1}, A_{2}$ as mentioned before and $A_{3}:=6 k \log k$ (by (18)) to obtain the following:

$$
\left|\alpha^{n-1} \cdot 10^{-(2 \ell+m)} \cdot\left(\frac{9 f_{k}(\alpha)}{a}\right)-1\right|>\exp \left(-9 \times 10^{12} k^{4} \log ^{2} k \log n\right),
$$

where we use that $1+\log k<3 \log k$ and $1+\log n<2 \log n$ hold for all $k \geq 2$ and $n \geq 4$, respectively. Comparing (16) and (20) and performing the respective calculations, we obtain the following:

$$
\ell<4 \times 10^{12} k^{4} \log ^{2} k \log n .
$$

3.2.2. An Inequality for $m$ in Terms of $k$

In light of (19) and (21), we deduce the following:

$$
h\left(\gamma_{3,2}\right)<10^{13} k^{4} \log ^{2} k \log n .
$$

This allows us to choose now $A_{3}:=10^{13} k^{5} \log ^{2} k \log n$. We then apply Matveev's theorem on (17) with the parameters $\gamma_{1}, \gamma_{2}$ and $\gamma_{3,2}$ to obtain the following:

$$
\left|\alpha^{n-1} \cdot 10^{-(\ell+m)} \cdot\left(\frac{9 f_{k}(\alpha)}{X}\right)-1\right|>\exp \left(-2 \times 10^{25} k^{8} \log ^{3} k \log ^{2} n\right) .
$$

Using now (17) and (22), we have the following:

$$
m<10^{25} k^{8} \log ^{3} k \log ^{2} n .
$$

3.2.3. An Inequality for $n$ in Terms of $k$

We finally use (21) and (23) combined with (10) to assert the following:

$$
\frac{n}{\log ^{2} n}<1.2 \times 10^{26} k^{8} \log ^{3} k
$$

In order to upper bound $n$ polinomially in terms of $k$, we use an analytical argument of Guzmán and Luca [34] who proved that if $m \geq 1, T>\left(4 m^{2}\right)^{m}$ and $T>x / \log ^{m} x$, then $x<2^{m} T \log ^{m} T$. In our case, we take $T:=1.2 \times 10^{26} k^{8} \log ^{3} k$ and $m:=2$ to obtain from (24) the following lemma.

Lemma 5. If $(n, k, a, b, \ell, m)$ is a solution of Equation (1) with $n \geq k+2$, then the following holds:

$$
2 \ell+m<n<5 \times 10^{30} k^{8} \log ^{5} k .
$$

\subsection{The Case of Large $k$}

Suppose that $k>430$. Note that for such values of $k$, we have the following:

$$
5 \times 10^{30} k^{8} \log ^{5} k<2^{k / 2} .
$$

Then, by Lemma 5, we obtain that the inequality $n<2^{k / 2}$ is satisfied when $k>430$, and therefore, we are in the hypothesis of Lemma 1. Applying the above lemma and Equation (1), we obtain the following:

$$
\left|\frac{a}{9} \cdot 10^{2 \ell+m} \cdot 2^{-(n-2)}-1\right|<\frac{3 \cdot 10^{\ell+m}}{2^{n-2}}+\frac{1}{2^{k / 2}}<\frac{30}{10^{\ell}}+\frac{1}{2^{k / 2}},
$$


where we have used that $10^{\ell+m} / 2^{n-2}<10 / 10^{\ell}$ (see (9)). Consequently, we have the following:

$$
\left|\frac{a}{9} \cdot 10^{2 \ell+m} \cdot 2^{-(n-2)}-1\right|<\frac{30}{2^{\theta \ell}}+\frac{1}{2^{k / 2}} \leq \frac{31}{2^{\lambda}}
$$

where $\theta:=(\log 10) /(\log 2)$ and $\lambda:=\min \{k / 2, \theta \ell\}$. Again, in order to use the result of Matveev, we take $t:=3$ and the following:

$$
\left(\gamma_{1}, b_{1}\right):=(a / 9,1), \quad\left(\gamma_{2}, b_{2}\right):=(10,2 \ell+m) \quad \text { and } \quad\left(\gamma_{3}, b_{3}\right):=(2,-(n-2)) .
$$

We begin by noticing that the three numbers $\gamma_{1}, \gamma_{2}, \gamma_{3}$ are positive rational numbers, so we can take $\mathbb{K}:=\mathbb{Q}$ for which $D:=1$. To see why the left-hand side of (25) is not zero, note that, otherwise, we would obtain $a \cdot 10^{2 \ell+m}=9 \cdot 2^{n-2}$, which is impossible since its left-hand side is divisible by 5 while its right-hand side is not.

Clearly, we can take $A_{1}:=\log 9, A_{2}:=\log 10$ and $A_{3}:=\log 2$. Here, we can also take $B:=n$. Then, Matveev's theorem together with a straightforward calculation gives the following:

$$
\left|\frac{a}{9} \cdot 10^{2 \ell+m} \cdot 2^{-(n-2)}-1\right|>\exp \left(-1.1 \times 10^{12} \log n\right),
$$

where we use $1+\log n<2 \log n$ holds for all $n \geq 4$. Comparing (25) and (26), taking logarithms and then performing the respective calculations, we arrive at the following:

$$
\lambda<1.8 \times 10^{12} \log n .
$$

Note that, if $\lambda=k / 2$, then $k<3.6 \times 10^{12} \log n$. Since $\log n<73 \log k$ holds for all $k>430$ by Lemma 5 , we obtain $k<2.7 \times 10^{14} \log k$, giving $k<10^{16}$. For the case when $\lambda=\theta \ell$, we have $\ell<5.5 \times 10^{11} \log n$. Here, proceeding as in (25), we obtain the following:

$$
\left|\frac{X}{9} \cdot 10^{\ell+m} \cdot 2^{-(n-2)}-1\right|<\frac{2^{\theta \ell}}{2^{n-2}}+\frac{2}{2^{k / 2}} \leq \frac{2^{k / 2}}{2^{k}}+\frac{2}{2^{k / 2}}=\frac{3}{2^{k / 2}} .
$$

The same argument used before also shows that the left-hand side of (27) is not zero. With a view toward applying Matveev's theorem, we take the same parameters as in the previous application, except by $\gamma_{1}$ and $b_{2}$, which, in this case, are given by $X / 9$ and $\ell+m$, respectively. As before, $\mathbb{K}:=\mathbb{Q}, D:=1, A_{2}:=\log 10, A_{3}:=\log 2$ and $B:=n$. Moreover, by (14), we have the following:

$$
h\left(\gamma_{1}\right)=\log X \leq(\ell+1) \log 10<1.3 \times 10^{12} \log n .
$$

Hence, we can take $A_{1}:=1.3 \times 10^{12} \log n$. This time, Matveev's theorem leads to the following:

$$
\exp \left(-6 \times 10^{23} \log ^{2} n\right)<\left|\frac{X}{9} \cdot 10^{\ell+m} \cdot 2^{-(n-2)}-1\right|<\frac{3}{2^{k / 2}},
$$

which implies $k<9.4 \times 10^{26} \log ^{2} k$. Hence, $k<5 \times 10^{30}$ and so, by Lemma 5, we obtain that $n<3.5 \times 10^{285}$. At this point, we summarize what we have obtained so far on the upper bounds for $k$ and $n$. The result is the following.

Lemma 6. If $(n, k, a, b, \ell, m)$ is a solution of Equation (1) with $k>430$ and $n \geq k+2$, then all inequalities hold:

$$
k<5 \times 10^{30} \text { and } 2 \ell+m<n<3.5 \times 10^{285}
$$

\subsection{Reducing the Bound on $k$.}

We now want to reduce our bound on $k$ by using Lemma 2. Let the following hold:

$$
\Gamma_{1}:=\log (a / 9)+(2 \ell+m) \log 10-(n-2) \log 2 .
$$


Then, from (25) we get that $\left|e^{\Gamma_{1}}-1\right|<31 / 2^{\lambda}$. Note that $31 / 2^{\lambda}<1 / 2$ whenever $\lambda \geq 6$. Now, assuming that $\lambda \geq 6$, we obtain $\left|e^{\Gamma_{1}}-1\right|<1 / 2$ and so Lemma 4 shows that $0<\left|\Gamma_{1}\right|<2\left|e^{\Gamma_{1}}-1\right|<62 / 2^{\lambda}$. Dividing the above inequality through $\log 2$ gives the following:

$$
0<\left|(2 \ell+m) \theta-n+\mu_{a}\right|<90 \cdot 2^{-\lambda} \quad \text { for all } \quad \lambda \geq 6,
$$

where $\mu_{a}:=2+(\log (a / 9)) /(\log 2)$. Taking $M:=3.5 \times 10^{285}$ we get that $2 \ell+m<M$. Applying now Lemma 2 to inequality (28) for each $a \in\{1,2, \ldots, 8\}$ we find, with the help of Mathematica, that $\lambda \leq 960$.

For the case $a=9$, we cannot use Lemma 2 because the corresponding value of $\epsilon$ is always negative. However, one can see that if $a=9$, then the resulting inequality from (28) has the following shape:

$$
|x \gamma-y|<90 \cdot 2^{-\lambda}
$$

with $\gamma:=\theta$ being an irrational number and $x:=2 \ell+m, y:=n-2 \in \mathbb{Z}$. In order to apply Lemma 3 on the left-hand side of (29), we define $\left[a_{0}, a_{1}, a_{2}, a_{3}, \ldots\right]=[3,3,9,2, \ldots]$ as the continued fraction of $\gamma$ and $p_{i} / q_{i}$ its $i$ th convergent. We can also take $M:=3.5 \times 10^{285}$ so that $x<M$ by Lemma 6. A quick inspection using Mathematica reveals that $q_{573} \leq M<q_{574}$ and therefore, $a_{M}:=\max \left\{a_{i} \mid 0 \leq i \leq 574\right\}=a_{135}=5393$. Hence, by Lemma 3, we obtain $|x \gamma-y|>1 /(5395(2 \ell+m))$, and after a comparison with (29), we obtain $\lambda \leq 967$. Thus, $\lambda \leq 967$ always holds.

Note that if $\lambda=k / 2$, then $k \leq 1934$. On the other hand, if $\lambda=\theta \ell$ then we have $\ell \leq 291$. Now, let the following hold:

$$
\Gamma_{2}:=(\ell+m) \log 10-(n-2) \log 2+\log (X / 9) .
$$

Here, (27) yields $\left|e^{\Gamma_{2}}-1\right|<3 / 2^{k / 2}$. Since $k>430$, we get that $\left|e^{\Gamma_{2}}-1\right|<1 / 2$. Using Lemma 4 again, we deduce that $0<\left|\Gamma_{2}\right|<6 / 2^{k / 2}$. Dividing through the above inequality by $\log 2$ gives the following:

$$
0<|(\ell+m) \theta-n+\mu(a, b, \ell)|<9 \cdot 2^{-k / 2},
$$

where $\mu(a, b, \ell):=2+(\log (X / 9) /(\log 2))$. Here, we also take $M:=3.5 \times 10^{285}$ and apply Lemma 2 to inequality (30) for all $a, b \in\{0,1, \ldots, 9\}$ with $a \geq 1, a \neq b$ and $1 \leq \ell \leq 291$, except when the following is true:

$$
(a, b, \ell) \in\{(1,0,1),(1,9,1),(2,0,1),(3,9,1),(4,0,1),(7,9,1),(8,0,1),(4,9,1),(5,0,1)\}
$$

and $(a, b, \ell)=(9,9, \ell)$ for all $\ell \geq 1$. Indeed, a computer search with Mathematica reveals that $k \leq 1955$. Now, we deal with the special cases mentioned just before. First of all, it is a straightforward exercise to check that in these cases, we have the following:

$$
\mu(a, b, \ell)=\left\{\begin{array}{cll}
2, & \text { if } & (a, b, \ell)=(1,0,1) \\
3, & \text { if } & (a, b, \ell)=(1,9,1),(2,0,1) \\
4, & \text { if } & (a, b, \ell)=(3,9,1),(4,0,1) \\
5, & \text { if } & (a, b, \ell)=(7,9,1),(8,0,1) \\
1+\theta, & \text { if } & (a, b, \ell)=(4,9,1),(5,0,1) \\
1+\ell \theta, & \text { if } & (a, b, \ell)=(9,9, \ell), \ell \geq 1 .
\end{array}\right.
$$

In these cases, the inequality (30) turns into the following:

$$
\begin{array}{cl}
|(m+1) \theta-(n-i)|<9 \cdot 2^{-k / 2} & (\text { for } i=2,3,4,5), \quad \text { or } \\
|(m+2) \theta-(n-1)|<9 \cdot 2^{-k / 2}, \quad \text { or } & |(2 \ell+m) \theta-(n-1)|<9 \cdot 2^{-k / 2} .
\end{array}
$$

In any case, by the same arguments used to get (29), we obtain $2^{k / 2}<1.7 \times 10^{290}$, which implies that $k \leq 1928$. Thus, $k \leq 1955$ holds for any choice of $\lambda$. Then, by Lemma 5 , $2 \ell+m<2.7 \times 10^{61}:=M$. With this new choice of $M$, Lemma 2 applied to inequality (28) 
implies that $\lambda \leq 222$ (including the case $a=9$ ). If $\lambda=k / 2$, then $k \leq 444$, while if $\lambda=\theta \ell$, we have that $\ell \leq 66$. We finally apply Lemma 2 with $M:=2.7 \times 10^{61}$ to inequality (30) for all $a, b \in\{0,1, \ldots, 9\}$ with $a \neq b, a \geq 1$ and $1 \leq \ell \leq 66$, except in the special cases mentioned above. With the help of Mathematica, we find that $k \leq 457$. The same upper bound for $k$ holds in the special cases. So, $k \leq 457$ holds for any choice of $\lambda$. Finally, taking $M:=8.2 \times 10^{55}$ and repeating the previous procedure, we obtain $k \leq 422$, which is a contradiction. Hence, the Equation (1) has no solutions for $k>430$.

\subsection{The Case of Small k}

Suppose now that $k \in[2,430]$. Note that for each of these values of $k$, Lemma 5 gives us absolute upper bounds for $n$. However, these upper bounds are so large and will be reduced by using Lemma 2 once again. To do this, we put the following:

$$
\Omega:=\left[\begin{array}{l}
\Omega_{1} \\
\Omega_{2}
\end{array}\right]=\left[\begin{array}{l}
(n-1) \log \alpha-(2 \ell+m) \log 10+\log \left(\left(9 f_{k}(\alpha)\right) / a\right) \\
(n-1) \log \alpha-(\ell+m) \log 10+\log \left(\left(9 f_{k}(\alpha)\right) / X\right)
\end{array}\right] .
$$

Thus, (16) and (17) can be rewritten as follows:

$$
\left|e^{\Omega_{1}}-1\right|<\frac{11}{10^{\ell}}, \quad \text { and } \quad\left|e^{\Omega_{2}}-1\right|<\frac{11}{10^{m}} .
$$

Now assuming $\ell \geq 2$ and $m \geq 2$, we see that $\left|e^{\Omega_{i}}-1\right|<1 / 2$ for all $i \in\{1,2\}$. Using Lemma 4 , we deduce the following:

$$
0<\left|\Omega_{1}\right|<22 / 10^{\ell} \quad \text { and } \quad 0<\left|\Omega_{2}\right|<22 / 10^{m} .
$$

Dividing both inequalities by $\log 10$, we obtain the following:

$$
\begin{gathered}
0<\left|(n-1) \tau_{1}-(2 \ell+m)+\mu_{1}(k, a)\right|<10 \cdot 10^{-\ell}, \\
0<\left|(n-1) \tau_{1}-(\ell+m)+\mu_{2}(k, \ell, a, b)\right|<10 \cdot 10^{-m},
\end{gathered}
$$

where

$$
\tau_{1}:=\frac{\log \alpha}{\log 10} \quad \text { and } \quad\left[\begin{array}{c}
\mu_{1}(k, a) \\
\mu_{2}(k, \ell, a, b)
\end{array}\right]:=\left[\begin{array}{c}
\frac{\log \left(\left(9 f_{k}(\alpha)\right) / a\right)}{\log 10} \\
\frac{\log \left(\left(9 f_{k}(\alpha)\right) / X\right)}{\log 10}
\end{array}\right] .
$$

Note that $\tau_{1}$ clearly is an irrational number because $\alpha$ and 10 are multiplicatively independent. Next, we shall apply Lemma 2 to (31) and (32). For this purpose, we put also $M_{k}:=5 \times 10^{30} k^{8} \log ^{5} k$, which is an upper bound on $n-1$ by Lemma 5 .

In the first application, we choose the following parameters:

$$
\tau:=\tau_{1}, \quad \mu:=\mu_{1}(k, a), \quad A:=10, \quad B:=10 .
$$

A computer search with Mathematica reveals that if $k \in[2,430]$ and $a \in\{1,2, \ldots, 9\}$, then the maximum value of $\lfloor\log (A q / \epsilon) / \log B\rfloor$ is 130 . Then, every possible solution $(n, k, a, b, \ell, m)$ of Equation (1) for which $(k, a) \in[2,430] \times[1,9]$ has $\ell \in[1,130]$.

For the second application, we take the following:

$$
\tau:=\tau_{1}, \quad \mu:=\mu_{2}(k, \ell, a, b), \quad A:=10, \quad B:=10 .
$$

In this case, Mathematica shows that for each $a, b \in\{0,1, \ldots, 9\}$ with $a \geq 1, a \neq b$, $k \in[2,430]$ and $\ell \in[1,130]$, the maximum value of $\lfloor\log (A q / \epsilon) / \log B\rfloor$ is 130 . Thus, $m \in[1,130]$ and so $n \in[1,2340]$.

Finally, we use Mathematica to display the values of $F_{n}^{(k)}$ for $(k, n) \in[2,430] \times[4,2340]$, and check that Equation (1) has only the solution listed in Theorem 1 (see Appendix A). This completes the analysis in the case $k \in[2,430]$ and ends the proof. 
Author Contributions: Proposing the problem, C.A.G.; conceptualization, J.L.H., J.J.B., C.A.G.; methodology, J.L.H., J.J.B., C.A.G.; investigation, J.L.H., J.J.B., C.A.G.; formal analysis, J.L.H.; writingreview and editing, J.J.B.; software, C.A.G. All authors have read and agreed to the published version of the manuscript.

Funding: Jhon J. Bravo was supported in part by Project VRI ID 5385, Universidad del Cauca, Colombia. Carlos A. Gómez was supported in part by Project 71280, Universidad del Valle, Colombia.

Acknowledgments: We thank the reviewers for their time spent on reviewing our manuscript and their comments helping us improving the manuscript. Jose L. Herrera thanks the Universidad del Cauca for support during his Ph.D. studies.

Conflicts of Interest: The authors declare no conflict of interest.

\section{Appendix A}

Below, we present the Mathematica commands used along the paper.

\section{Appendix A.1. Basic Commands}

First, we present the commands to approximate the dominant $\operatorname{root} \alpha$ of $\Psi_{k}(x)$ and the commands to display the first $n$ generalized Fibonacci numbers and the largest prime factor of $n$. We also give a curious number function.

- The dominant root $\alpha$ of $\Psi_{k}(x)$ :

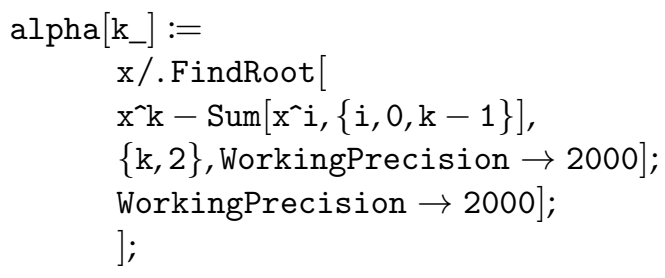

- $\quad$ The first $n$ generalized Fibonacci numbers:

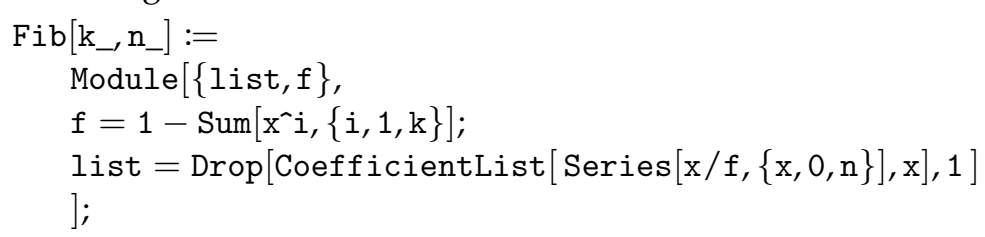

- $\quad$ The largest prime factor of $n$ :

LargestPrimeFactor $\left[\mathrm{n}_{-}\right]:=\operatorname{Max}[$ FactorInteger $[\mathrm{n}][[\mathrm{All}, 1]]]$;

- A curious number of the form $\underbrace{a \cdots a}_{\ell} \underbrace{b \cdots b}_{m} \underbrace{a \cdots a}_{\ell}$ :

$$
\begin{aligned}
& \text { Courios[a_, } \left.\mathrm{b}_{-}, \mathrm{l}_{\boldsymbol{1}^{\prime}} \mathrm{m}_{-}\right] \\
& \quad:=(1 / 9) *\left(\mathrm{a} * 10^{-}((2 * \mathrm{l})+\mathrm{m})-(\mathrm{a}-\mathrm{b}) * 10^{\wedge}(1+\mathrm{m})+(\mathrm{a}-\mathrm{b}) * 10^{\wedge}(1)-\mathrm{a}\right)
\end{aligned}
$$

\section{Appendix A.2. Basic Algorithms}

We next give the Mathematica algorithm used to determine all powers of two, which are curious numbers, and the algorithm employed in s Section 3.5 to find all the solutions in the small range of $k$.

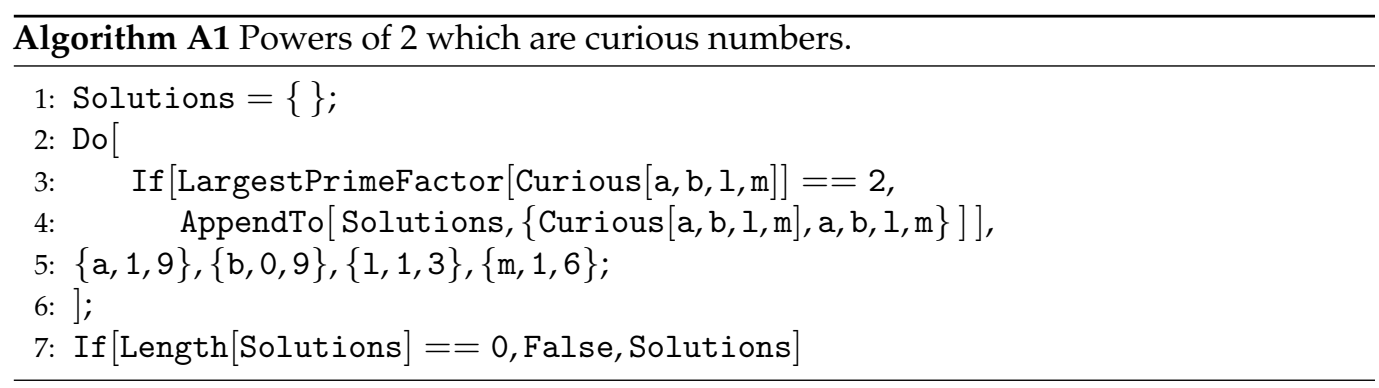




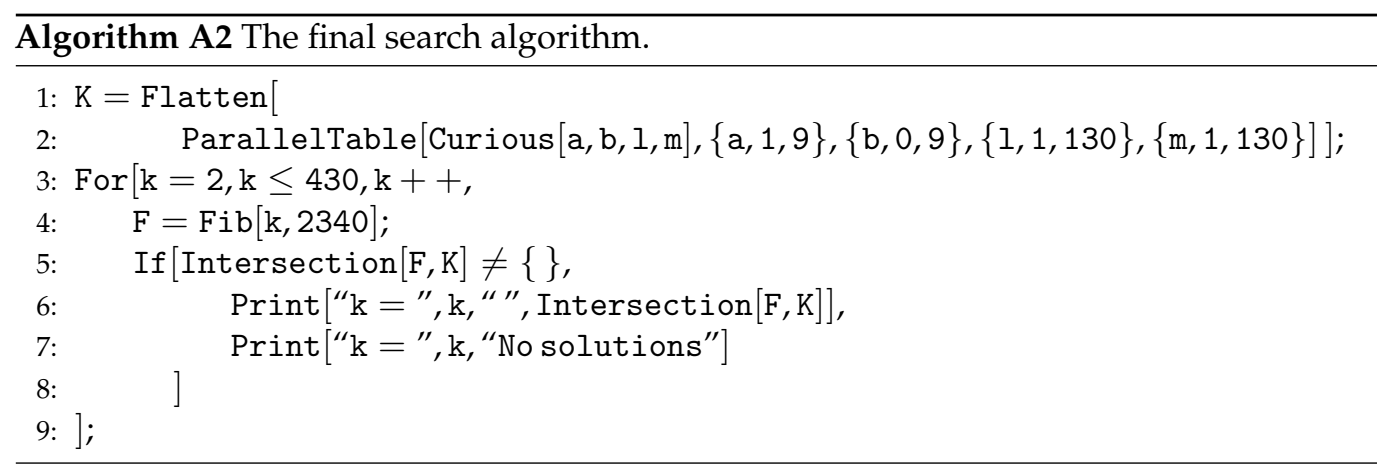

\section{References}

1. The On-Line Encyclopedia of Integer Sequences (OEIS). Available online: http:/ / oeis.org (accessed on 20 August 2021).

2. $\quad$ Borade N.; Mayle, J. Square Curious Numbers. J. Integer Seq. 2021, 41, 21.7.4.

3. Luca, F. Fibonacci and Lucas numbers with only one distinct digit. Port. Math. 2020, 57, 243-254.

4. Erduvan, F.; Keskin, R. Repdigits as products of two Fibonacci or Lucas numbers. Proc. Math. Sci. 2020, 130, 14. [CrossRef]

5. Faye, B.; Luca, F. Pell and Pell-Lucas numbers with only one distinct digits. Ann. Math. 2015, 45, 55-60.

6. Normenyo, B.; Luca, F.; Togbé. A. Repdigits as sums of three Pell numbers. Per. Math. Hung. 2018, 77, 318-328. [CrossRef]

7. Normenyo, B.; Luca, F.; Togbé, A. Repdigits as sums of four Pell numbers. Bol. Soc. Mat. Mex. 2019, 25, $249-266$.

8. Marques, D. On k-generalized Fibonacci numbers with only one distinct digit. Util. Math. 2015, 98, $23-31$.

9. Bravo, J.J.; Luca, F. On a conjecture about repdigits in k-generalized Fibonacci sequences. Publ. Math. Debr. 2013, 82, 623-639. [CrossRef]

10. Alahmadi, A.; Altassan, A.; Luca, F.; Shoaib H. Products of k-Fibonacci numbers which are rep-digits. Publ. Math. Debr. 2020, 97, 1-15. [CrossRef]

11. Bravo, E.F.; Gómez, C.A.; Luca, F. Products of consecutive Tribonacci numbers with only one distinct digit. J. Integer Seq. 2019, 22, 19.6.3.

12. Marques, D.; Togbé, A. On repdigits as product of consecutive Fibonacci numbers. Rend. Istit. Mat. Univ. Trieste. $2012,44,393-397$.

13. Bravo, J.J.; Herrera, J.L. Repdigits in generalized Pell sequences. Arch. Math. 2020, 56, 249-262. [CrossRef]

14. Bravo, J.J.; Luca, F. Repdigits as sums of two k-Fibonacci numbers. Monatsh Math. 2015, 176, 31-51. [CrossRef]

15. Coufal, P.; Trojovský, P. Repdigits as Product of Terms of $k$-Bonacci Sequences. Mathematics 2021, 9, 682. [CrossRef]

16. Bravo, J.J.; Luca, F. Powers of two in generalized Fibonacci sequences. Rev. Colomb. Mat. 2012, 46, 67-79.

17. Bravo, J.J.; Luca, F. Coincidences in generalized Fibonacci recurrences. J. Number Theory 2013, 133, 2121-2137. [CrossRef]

18. Alahmadi, A.; Altassan, A.; Luca, F.; Shoaib H. $k$-generalized Fibonacci numbers which are concatenations of two repdigits. Glas. Mat. 2021, 56, 29-46. [CrossRef]

19. Trojovský, P. Fibonacci numbers with a prescribed block of digits. Mathematics 2020, 8, 639-645. [CrossRef]

20. Bravo, E.F.; Bravo, J.J.; Gómez, C.A. $k$-Fibonacci numbers with two blocks of repdigits. Math. Slovaca 2020, 71, 267-274. [CrossRef]

21. Everest, G.; Van der Poorten, A.; Shparlinski, I.; Ward, T. Recurrence Sequences; American Mathematical Society: Providence, RI, USA, 2003; Volume 104.

22. Hua, L.K.; Wang, Y. Applications of Number Theory to Numerical Analysis; Springer: Berlin/Heidelberg, Germany, 1981.

23. Wolfram, D.A. Solving generalized Fibonacci recurrences. Fibonacci Quart. 1998, 36, 129-145.

24. Miles, E.P., Jr. Generalized Fibonacci numbers and associated matrices. Am. Math. Monthly 1960, 67, 745-752. [CrossRef]

25. Miller, M. D. Mathematical Notes: On Generalized Fibonacci Numbers. Am. Math. Monthly 1971, 78, 1108-1109. [CrossRef]

26. Dresden, G.P.; Du, Z. A simplified Binet formula for $k$-generalized Fibonacci numbers. J. Integer Seq. 2014, 17, 14.4.7.

27. Bravo, J.J.; Gómez, C.A.; Luca, F. Powers of two as sums of two k-Fibonacci numbers. Miskolc Math. Notes 2016, 17, 85-100. [CrossRef]

28. Bravo, J.J.; Gómez, C.A.; Luca, F. A Diophantine equation in k-Fibonacci numbers and repdigits. Colloq. Math. 2018, 152, $299-315$. [CrossRef]

29. Bravo, J.J.; Gómez, C.A.; Herrera J.L. On the intersection of k-Fibonacci and Pell numbers. Bull. Korean Math. Soc. 2019, 56, 535-547.

30. Matveev, E.M. An explicit lower bound for a homogeneous rational linear form in the logarithms of algebraic numbers II. Izv. Ross. Akad. Nauk Ser. Mat. 2020, 64, 1217-1269. [CrossRef]

31. Dujella, A.; Pethő, A. A generalization of a theorem of Baker and Davenport. Q. J. Math. 1998, 49, 291-306. [CrossRef]

32. Murty, RM.; Esmonde, J. Problems in Algebraic Number Theory, 2rd ed.; Springer: Berlin/Heidelberg, Germany, 2005 ; Volume 190.

33. Bravo, J.J.; Herrera J.L.; Luca, F. Common values of generalized Fibonacci and Pell sequences. J. Number Theory 2021, $226,51-71$. [CrossRef]

34. Guzmán, S.; Luca, F. Linear combinations of factorials and S-units in a binary recurrence sequence. Ann. Math. Québec 2014, 38, 169-188. 